\title{
Remote sensing image multi-feature fusion based on NSCT transform and compressive sensing
}

\author{
Zhiliang $\mathrm{Wu}^{\mathrm{a}}{ }^{\text {, Yongdong Huang }}{ }^{\mathrm{b}}$ and Kang Zhang ${ }^{\mathrm{c}}$
}

Institute of image processing and understanding, Beifang University of Nationalities, Yinchuan 750021, China;

awzlwlm@163.com, bhuang_yongdong@163.com, 'chang_kangkk@163.com

Keywords: Remote sensing image fusion, NSCT transform, compressive sensing, multi-feature fusion.

\begin{abstract}
For the remote sensing image fusion algorithm based on non-subsampled Contourlet transform (NSCT) calculation complexity and cannot extract details from source images effectively. So a new remote sensing image multi-feature fusion algorithm based on NSCT transform and compressive sensing is proposed. Firstly, luminance component $\mathrm{V}$ of multi-spectral image is extracted via HSV transform, and decompose the luminance component V and PAN image by NSCT; Then, Apply PCNN-based fusion rules to fusing low frequency sub-band, and the equivalent number is utilized as the linking strength; For the high frequency sub-band, a fusion rule based on the multi-feature in compressive sensing domain is presented; Finally, the fused images are obtained by inverse NSCT transform and inverse HSV transform, respectively. The experimental results show that the proposed fusion algorithm substantially outperforms the best-known remote sensing image fusion algorithms in terms of calculation complexity and the quality of the fused image, and has the better performance in visual effect and objective evaluation metrics
\end{abstract}

\section{Introduction}

In recent years, fusion technology of MS image and PAN image plays a importance role in the field of military and civilian [1]. MS image has high spectral resolution, but low spatial resolution; the spatial resolution of PAN image is high while the spectral resolution is low. Therefore, combine the spectral information of MS image and the spatial information of PAN image, which is conducive to achieve a comprehensive description of the scene and improve the observer's understanding of the image.

At present, the multi-solution analysis-based algorithm has become the mainstream of the MS image and PAN image fusion algorithm. One of the NSCT transform as multi-solution analysis algorithm [2], it not only inherit the anisotropy and localization characteristics, but also has the shift-invariance property. Thus, the NSCT is more suitable for image fusion. Unfortunately, the NSCT-based image fusion methods have a higher computational complexity of high frequency sub-band, and poor real-time performance. In order to solve this kind of problem effectively, many researchers have applied compressive sensing (CS) theory to image fusion which based NSCT transform [3-6]. However, these methods using only a single fusion rule in high-frequency sub-band, for example, literature [3] used weighted standard deviation-based fusion rule, literature [4-5] adopted maximizing neighborhood average gradient, etc. When using these single characteristics fusion rule in the fusion image, it will lead to optimization of visual and objective evaluation indexes can not be realized [2].

\section{Proposed Fusion Algorithm}

The low-frequency coefficient of image after NSCT decomposition contains approximation image, reflecting the general characteristics of the original image, the high frequency coefficient of image after NSCT decomposition contains the image details and edge feature information. Therefore, in the 
remote sensing image fusion method based on multi-solution analysis, the selection of fusion rules play an important role in image fusion quality.

\subsection{Fusion rule of low-frequency sub-band}

The low-frequency coefficient of image after NSCT decomposition contains approximation image, reflecting the general characteristics of the original image. In this paper, apply based on PCNN fusion rule to better fusion low-frequency coefficients, and the equivalent number of looks is utilized as the linking strength.

For image $I, I(i, j)$ is pixel value at $(i, j)$, and the equivalent number is defined as:

$$
m(i, j)=\frac{\bar{I}(i, j)^{2}}{\operatorname{Std}[I(i, j)]^{2}},
$$

where $\bar{I}(i, j)$ and $\operatorname{Std}[I(i, j)]$ represent the regional mean value and regional standard deviation of the image $I$ respectively, In this paper the size of the regional is $3 \times 3$.

\subsection{Fusion rule of high-frequency sub-band}

The high frequency coefficient of image after NSCT decomposition contains the image details and edge feature information. Therefore, the suitable high frequency fused rules is of great significance to improve the quality of the fused image. Commonly, the high frequency coefficient is often adopted the average gradient based fusion rules, the variance based fusion rules, the energy based fusion rules, and the other characteristics based fusion rules. However, when the above single characteristics based fusion rule is used in the fusion image, it will lead to optimization of visual and objective evaluation indexes cannot be realized. In order to solve this problem, a fusion rule based on the multi-feature in compressive sensing domain is proposed .The process is as follows:

Step1 Apply the measurement matrix to measure the high frequency subband of $\mathrm{V}$ component and the PAN image, and obtain the measured value.

Step2 Calculate the average gradient $\operatorname{Grad}_{P A N}, \operatorname{Grad}_{V}$, standard deviation $S D_{P A N}, S D_{V}$ and energy $E_{P A N}$, $E_{V}$ of V component and PAN image respectively.

Step3 Calculate the average gradient $\operatorname{Grad}_{P A N}(i, j), \operatorname{Grad}_{V}(i, j)$, regional standard deviation $S_{P A N}(i, j)$, $S D_{V}(i, j)$ and regional energy $E_{P A N}(i, j), E_{V}(i, j)$ of measured values of the $\mathrm{V}$ component and the PAN image respectively. In this paper the size of the regional is $3 \times 3$.

Step4 Calculate the relative average gradient, relative standard deviation and relative energy respectively

$$
\begin{aligned}
& K_{1}(i, j)=\frac{\operatorname{Grad}_{P A N}(i, j) / \operatorname{Grad}_{P A N}}{\operatorname{Grad}_{V}(i, j) / \operatorname{Grad}_{V}}, \\
& K_{2}(i, j)=\frac{S D_{P A N}(i, j) / S D_{P A N}}{S D_{V}(i, j) / S D_{V}}, \\
& K_{3}(i, j)=\frac{E_{P A N}(i, j) / E_{P A N}}{E_{V}(i, j) / E_{V}} .
\end{aligned}
$$

If $\operatorname{Grad}_{V}(i, j), S D_{V}(i, j)$ and $E_{V}(i, j)$ exist equal to 0 , then considering the features is the worst, secondly calculate the ratio of the remaining features; If $\operatorname{Grad}_{V}(i, j)=S D_{V}(i, j)=E_{V}(i, j)=0$, the maximum absolute value is used as the final fusion rule.

Step 5 Compare the following three kinds of ratios of measurement value of decision fusion rules

$$
\begin{aligned}
& R_{1}(i, j)=\left\{\begin{array}{c}
K_{1}(i, j), K_{1}(i, j) \geq 1 \\
1 / K_{1}(i, j), K_{1}(i, j)<1
\end{array},\right. \\
& R_{2}(i, j)=\left\{\begin{array}{c}
K_{2}(i, j), K_{2}(i, j) \geq 1 \\
1 / K_{2}(i, j), K_{2}(i, j)<1
\end{array},\right. \\
& R_{3}(i, j)=\left\{\begin{array}{c}
K_{3}(i, j), K_{3}(i, j) \geq 1 \\
1 / K_{3}(i, j), K_{3}(i, j)<1
\end{array} .\right.
\end{aligned}
$$

For any of the measure values, the fusion rule corresponding to the maximum ratio is chosen as the final fusion rule at this measure values. 
Step6 Apply sparsity adaptive matching pursuit(SAMP) algorithm to reconstruct the fused high-frequency subband.

\subsection{Fusion rule of high frequency subband}

Based on the above analysis, the algorithm steps are as follows:

Step1 Extract the luminance component V of MS image via HSV transform, and perform histogram matching on the PAN image;

Step2 Perform the NSCT transform on the PAN image after histogram matching and the V component of MS image respectively;

Step3 Use the fused rules described in section 2.1 to fuse the low frequency subband of the PAN image and the $\mathrm{V}$ component of MS image.

Step4 Use the fused rules described in section 2.2 to fuse the high frequency subband of the PAN image and the V component of MS image.

Step5 Obtain the fused image by inverse NSCT transform and inverse HSV transform.

The diagram of the proposed algorithm is shown in Fig1.

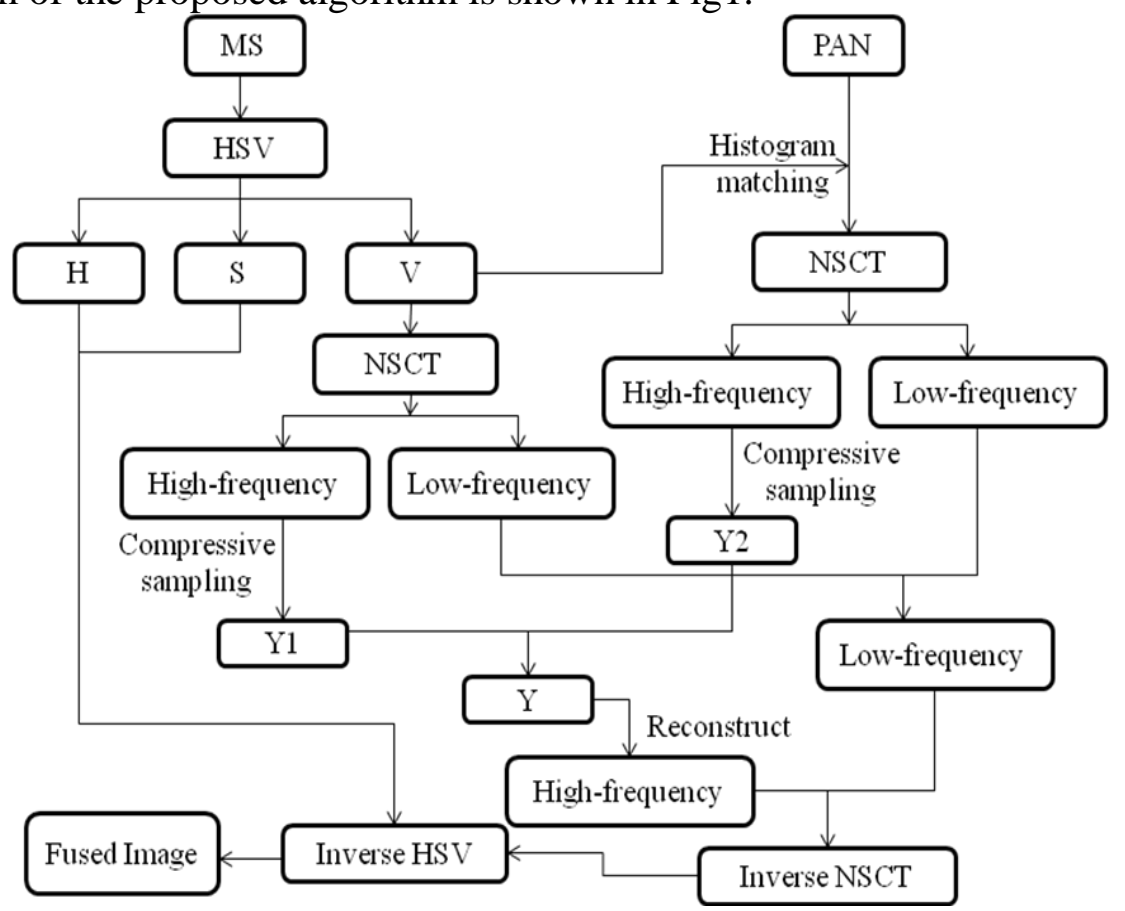

Fig. 1. Schematic diagram of the proposed image method

\section{Experimental results and analysis}

In order to verify the validity and superiority of the remote sensing image fusion algorithm proposed in this paper, we use Matlab7.1 as the tool to select two different remote sensing images for simulation experiment and analysis, and compare with the literature [2], [4] [5] and the fusion method used in the high frequency reconstruction using the OMP algorithm (orthogonal matching tracking algorithm) as "NSCT-OMP". Among them, NSCT transform adopts "9-7" filter and "pkva" filter, the direction series is [2, 3, 4]. The parameters of the method PCNN are: $\alpha_{L}=1, \alpha_{\theta}=0.2$, $W=[0.707,1,0.707 ; 1,0,1 ; 0.707,1,0.707], V_{L}=1, V_{\theta}=20, n=20$. The Two sets of MS images and PAN images, as well as the use of different fusion algorithm fusion results shown in Figure 2 and Figure 3.

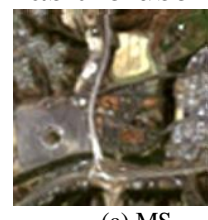

(a) MS

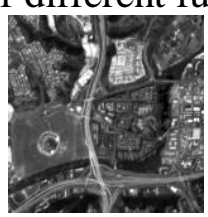

(b) PAN

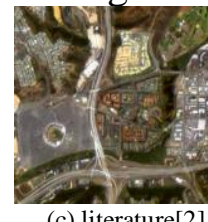

(c) literature[2]

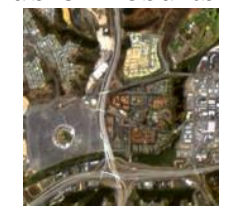

(d) literature[4]

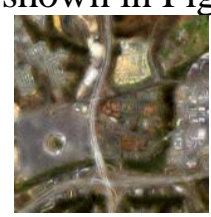

(e) literature[5]

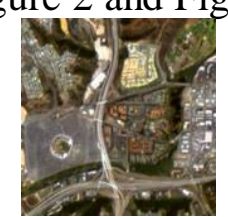

f) NSCT-OMP (g) proposed method

Fig. 2 images for team one and the fused results using various methods 


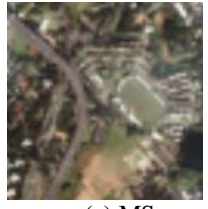

(a) MS

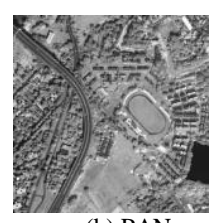

(b) PAN
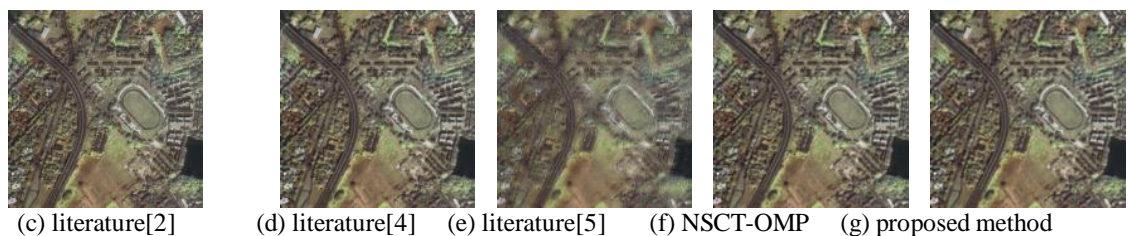

$\begin{array}{llll}\text { (d) literature[4] } & \text { (e) literature[5] } & \text { (f) NSCT-OMP } & \text { (g) proposed method }\end{array}$

Fig. 3 images for team two and the fused results using various methods

The purpose of MS image fusion with PAN image is to preserve the spatial resolution of the PAN image and the spectral resolution of the MS image as much as possible. Therefore, this paper mainly uses Mean, Information Entropy (EI), Deviation Index (DC), Correlation Coefficient (CC), Root Mean Square Error (RMSE), Peak Signal to Noise Ratio (PSNR), Structural Similarity (SSIM) and Relative to the overall dimension of comprehensive error (ERGAS) 8 kinds of objective evaluation of the quantitative analysis of the results of the fusion analysis and comparison. The fusion results of the two sets of MS images and PAN images using different fusion algorithms are shown in Table 1 and Table 2.

Table 1. Objective evaluation results of the first group of images

\begin{tabular}{cccccccccc}
\hline Method & Band & Mean & EI & PSNR & RMSE & SSIM & CC & DC & ERGAS \\
\hline \multirow{2}{*}{ Literature } & $\mathrm{R}$ & 108.588 & 7.463 & 39.767 & 13.639 & 0.735 & 0.736 & & \\
{$[2]$} & $\mathrm{G}$ & 98.774 & 7.432 & 41.330 & 12.515 & 0.764 & 0.766 & 0.255 & 6.560 \\
& $\mathrm{~B}$ & 78.290 & 7.432 & 45.547 & 10.136 & 0.854 & 0.856 & & \\
\hline \multirow{2}{*}{ Literature } & $\mathrm{R}$ & 108.586 & 7.515 & 39.468 & 13.844 & 0.737 & 0.738 & & \\
{$[4]$} & $\mathrm{G}$ & 98.781 & 7.485 & 41.189 & 12.703 & 0.765 & 0.766 & 0.255 & 6.660 \\
& $\mathrm{~B}$ & 78.527 & 7.460 & 45.390 & 10.296 & 0.853 & 0.854 & & \\
\hline \multirow{2}{*}{ Literature } & $\mathrm{R}$ & 54.510 & 6.624 & 18.436 & 25.484 & 0.459 & 0.702 & & \\
{$[5]$} & $\mathrm{G}$ & 49.587 & 6.560 & 19.729 & 23.597 & 0.472 & 0.732 & 0.483 & \multirow{2}{*}{12.521} \\
& $\mathrm{~B}$ & 39.421 & 6.512 & 22.734 & 20.181 & 0.525 & 0.828 & & \\
\multirow{2}{*}{ NSCT- } & $\mathrm{R}$ & 108.576 & 7.513 & 39.647 & 13.721 & 0.741 & 0.741 & & \\
OMP & $\mathrm{G}$ & 98.777 & 7.483 & 41.368 & 12.590 & 0.768 & 0.769 & 0.254 & 6.599 \\
& $\mathrm{~B}$ & 78.524 & 7.458 & 45.592 & 10.193 & 0.855 & 0.856 & & \\
\hline \multirow{2}{*}{ proposed } & $\mathrm{R}$ & 108.572 & 7.507 & 39.911 & 13.541 & 0.746 & 0.746 & & \\
method & $\mathrm{G}$ & 98.773 & 7.478 & 41.632 & 12.425 & 0.773 & 0.774 & 0.250 & 6.510 \\
& $\mathrm{~B}$ & 78.521 & 7.455 & 45.879 & 10.047 & 0.859 & 0.860 & & \\
\hline
\end{tabular}

Table 2. Objective evaluation results of the second group of images

\begin{tabular}{cccccccccc}
\hline Method & Band & Mean & EI & PSNR & RMSE & SSIM & CC & DC & ERGAS \\
\hline \multirow{2}{*}{ Literature } & $\mathrm{R}$ & 109.967 & 7.131 & 36.429 & 14.409 & 0.492 & 0.494 & & \\
{$[2]$} & $\mathrm{G}$ & 103.744 & 7.140 & 37.141 & 13.723 & 0.513 & 0.513 & 0.309 & 6.502 \\
& $\mathrm{~B}$ & 87.941 & 6.809 & 39.951 & 11.659 & 0.513 & 0.516 & & \\
\hline \multirow{2}{*}{ Literature } & $\mathrm{R}$ & 109.963 & 7.170 & 38.326 & 14.370 & 0.504 & 0.505 & & \\
{$[4]$} & $\mathrm{G}$ & 103.776 & 7.178 & 38.972 & 13.691 & 0.525 & 0.525 & 0.282 & 6.484 \\
& $\mathrm{~B}$ & 87.922 & 6.845 & 41.252 & 11.620 & 0.526 & 0.528 & & \\
\hline \multirow{2}{*}{ Literature } & $\mathrm{R}$ & 55.201 & 6.404 & 17.228 & 25.420 & 0.292 & 0.436 & & \\
{$[5]$} & $\mathrm{G}$ & 52.092 & 6.393 & 18.525 & 23.515 & 0.323 & 0.461 & 0.477 & \multirow{2}{*}{11.350} \\
& $\mathrm{~B}$ & 44.137 & 6.090 & 20.302 & 20.525 & 0.304 & 0.455 & & \\
\multirow{2}{*}{ NSCT- } & $\mathrm{R}$ & 109.959 & 7.155 & 38.557 & 14.205 & 0.510 & 0.512 & & \\
OMP & $\mathrm{G}$ & 103.775 & 7.167 & 39.205 & 13.532 & 0.531 & 0.531 & 0.308 & 6.408 \\
& $\mathrm{~B}$ & 87.922 & 6.832 & 41.408 & 11.481 & 0.532 & 0.534 & & \\
\hline \multirow{2}{*}{ proposed } & $\mathrm{R}$ & 109.963 & 7.117 & 38.970 & 13.971 & 0.518 & 0.521 & & \\
method & $\mathrm{G}$ & 103.774 & 7.136 & 39.619 & 13.309 & 0.539 & 0.539 & 0.303 & 6.301 \\
& $\mathrm{~B}$ & 87.923 & 6.799 & 41.759 & 11.280 & 0.540 & 0.544 & & \\
\hline
\end{tabular}

\section{Summary}

Based on the NSCT transform multi-directions, time-frequency localization, and the basis of the characteristic of CS theory can reduce the amount of calculation, the paper presents a multi-feature 
remote sensing image fusion method based on NSCT and CS. Apply PCNN-based fusion rules to fusing low frequency sub-band, and equivalent number of the low frequency sub-band as PCNN link strength; For high frequency sub-band, this paper proposes a multi-feature fusion rule based on CS; Through two groups of MS and PAN image fusion experiments, it shows that this method can reduce computational complexity and can improve the quality of image, and it has good performance on the visual effects and objective indexes .

\section{Acknowledgements}

This work is supported by National Natural Science Foundation of China (Grant. No 61261043), and Research Project of Beifang University of Nationalities(Grant. No2016GQR02).

\section{References}

[1]. Brunner D, Lemoine G, Bruzzone L. Earthquake damage assessment of buildings using VHR optical and SAR imagery. IEEE Transactions on Geoscience and Remote Sensing .Vol. 48 (2010) No. 5, p. 2403-2420.

[2]. Wang F, Cheng Y M, Li S. et. al. Remote sensing image fusion algorithm based on multi-feature. Journal of Northwestern Polytechnical University. Vol. 33 (2015) No. 3, p. 489-494.

[3]. Xing Y Q, Wang X D and Bi K et. al. Fusion technique for images based on non-subsampled Contourlet transform and compressive sensing. Control and Decision. Vol. 29 (2014) No. 4, p. 585-592.

[4]. Wu Y H, Yan D and Ma M X, et al. An improved compressive sensing image fusion algorithm based on NSCT transform[J]. Applied Mechanics and Materials. Vol. 433 (2013) No. 435, p. 306-309.

[5]. Xing X X, Liu F and Shang W W, et al. Medical image fusion in compressed sensing based on non-subsampled Contourlet transform//Proceedings of the 9th IEEE International Conference on Mobile Ad-hoc and Sersor Networks. Los Alamitos: IEEE Computer Society Press. (2013), p. 490-493.

[6]. Yin M, Pang J Y and Wei Y Y et. al. Image fusion algorithm based on nonsubsampled dual-tree complex Contourlet transform and compressive sensing pulse coupled neural network. Journal of Computer-Aided Design \& Computer Graphics. Vol. 28 (2016) No. 3, p. 411-419. 